\title{
Exploring Abnormal Behavior Patterns of Online Users With Emotional Eating Behavior: Topic Modeling Study
}

Youjin Hwang ${ }^{1}$, PhD; Hyung Jun Kim ${ }^{1}$, MA; Hyung Jin Choi ${ }^{2}$, MD, PhD; Joonhwan Lee ${ }^{1}$ PhD

${ }^{1}$ Human Computer Interaction \& Design Lab, Seoul National University, Seoul, Republic of Korea

${ }^{2}$ Functional Anatomy of Metabolism Regulation Lab, Seoul National University College of Medicine, Seoul, Republic of Korea

Corresponding Author:

Joonhwan Lee, $\mathrm{PhD}$

Human Computer Interaction \& Design Lab

Seoul National University

64-411, Seoul National University

Gwanakro 1

Seoul, 08826

Republic of Korea

Phone: 8228806450

Email: joonhwan@snu.ac.kr

\section{Abstract}

Background: Emotional eating (EE) is one of the most significant symptoms of various eating disorders. It has been difficult to collect a large amount of behavioral data on EE; therefore, only partial studies of this symptom have been conducted. To provide adequate support for online social media users with symptoms of EE, we must understand their behavior patterns to design a sophisticated personalized support system (PSS).

Objective: This study aimed to analyze the behavior patterns of emotional eaters as the first step to designing a personalized intervention system.

Methods: The machine learning (ML) framework and Latent Dirichlet Allocation (LDA) topic modeling tool were used to collect and analyze behavioral data on EE. Data from a subcommunity of Reddit, /r/loseit, were analyzed. This dataset included all posts and feedback from July 2014 to May 2018, comprising 185,950 posts and 3,528,107 comments. In addition, deleted and improperly collected data were eliminated. Stochastic gradient descent-based ML classifier with an accuracy of $90.64 \%$ was developed to collect refined behavioral data of online users with EE behaviors. The expert group that labeled the dataset to train the ML classifiers included a medical doctor specializing in EE diagnosis and a nutritionist with profound knowledge of EE behavior. The experts labeled 5126 posts as EE (coded as 1) or others (coded as 0 ). Finally, the topic modeling process was conducted with LDA.

Results: The following 4 macroperspective topics of online EE behaviors were identified through linguistic evidence regarding each topic: addressing feelings, sharing physical changes, sharing and asking for dietary information, and sharing dietary strategies. The 5 main topics of feedback were dietary information, compliments, consolation, automatic bot feedback, and health information. The feedback topic distribution significantly differed depending on the type of EE behavior (overall $P<.001$ ).

Conclusions: This study introduces a data-driven approach for analyzing behavior patterns of social website users with EE behaviors. We discovered the possibility of the LDA topic model as an exploratory user study method for abnormal behaviors in medical research. We also investigated the possibilities of ML- and topic modeling-based classifiers to automatically categorize text-based behavioral data, which could be applied to personalized medicine in future research.

(J Med Internet Res 2020;22(3):e15700) doi: 10.2196/15700

\section{KEYWORDS}

emotional eating; eating disorder; machine learning; data-driven research; behavior analysis; topic modeling; Latent Dirichlet Allocation 


\section{Introduction}

\section{Background}

A large population is affected by eating disorders, including anorexia nervosa, bulimia nervosa, and binge eating disorder. According to statistics from the UK Addiction Treatment Centers, about 725,000 people in Britain have been affected by eating disorders, whereas others put the number as high as 1.6 million. The actual population that is affected by eating disorders is predicted to be even higher, considering the fact that many hesitate to seek professional help [1].

One of the common symptoms of eating disorders is emotional eating (EE), which is defined as "eating in response to any emotion, whether that be positive or negative" [2,3]. People with EE behavior frequently consume large quantities of comfort food that are usually nutritionally imbalanced, as a response to feelings instead of hunger [3,4]. The investigation of EE behavior is critical as many people with EE behaviors easily transition into those with serious eating disorders [5].

Few studies with a large amount of data were conducted regarding EE behavior. Analyzing the large quantity of behavioral data in diet-related posts on social media could be the first step in designing a support system for social media users. Previous studies in the field of human-computer interaction (HCI) have analyzed the users' behaviors through the utilization of data-driven technologies [6-10]. These studies analyze various user-created contents such as texts, images, or other categories of user logs [11-13]. Meaningful information can be extracted from these data, increasing the number of studies that utilize such a large amount of data to analyze the characteristics and patterns of abnormal behaviors [14-17].

When it comes to health-related abnormalities, face-to-face personal interviews have been used as the traditional method of behavioral analysis. However, people in the predisease state rarely visit experts because of the low perceived severity of their status, and thus, it is difficult to collect the data from people in the predisease state [17]. This limited dataset can result in biased results because of the unique characteristics of the condition of each group. Moreover, data from face-to-face interaction during a short period of time are not natural data collected from daily activities [18]. Therefore, observing the subjects in their natural environment through social media scanning is a promising alternative method for abnormal behavior analysis $[19,20]$. Previous studies have already used the information on social media to detect depression and insomnia [21-26].

Previous studies used natural language processing (NLP) technology to recognize certain behavioral patterns in social media and determined abnormal behaviors with repeated use of keywords or synonyms in the online posts [27-30]. For example, De Choudhury et al [23] used the Reddit community data to predict individuals who are more likely to undergo transitions from mental health discourse to suicidal ideation. They analyzed the linguistic structures such as the fraction of nouns, verbs, and adverbs used in posts and comments.
In this study, we used Latent Dirichlet Allocation (LDA), which is a high-performance topic modeling tool [31-33]. Compared with traditional text analysis methods such as interpretivist text analysis and systematic qualitative coding, LDA can capture unusual structures that exist in the natural language data as it is based on unsupervised learning, which can perform more complex tasks [34].

\section{Objective}

This study aimed to investigate the online behavior patterns of emotional eaters using the topic modeling method. We collected posts $(n=185,950)$ and comments $(n=3,528,107)$ from $/ r / l o s e i t$, a subcommunity of Reddit, an online social forum, and classified these posts and comments with a machine learning (ML) framework. LDA [32] was used to examine the behavior patterns of emotional eaters. The feedback on the EE posts was also classified into multiple topics by LDA. We also compared the different proportions of feedback topics on each behavior topic to understand the interactions in the comments section. On the basis of these findings, we discussed the design implications for a personalized supporting system in health care.

\section{Methods}

\section{Data Collection}

We used open-source data distributed through Google BigQuery, which originated from Reddit, one of the biggest online social news websites and forums. Contents in Reddit are organized in subcommunities by areas of interests, called subreddits. Among these subreddits, /r/loseit is one of the biggest subreddit community that deals with weight management. In /r/loseit, the user-generated content comprises various topics related to obesity and weight loss, such as personal experiences; recommendations; and reviews of medications, medical procedures, diets, and exercises [35]. For research ethics, to protect personal information, we did not collect any information that can be used to identify the specific users, such as personal ID and name. When including quotes in this paper, we paraphrased all sentences to remove words that can identify the individual or allow searching of the post. With this process, we constructed a dataset that included all posts and feedback between July 2014 and May 2018, comprising 185,950 posts and 3,528,107 comments. Deleted and improperly collected data were eliminated.

\section{Process of Data Analysis}

\section{Data Labeling With Expert Group}

To classify data from the area of interest from the whole dataset, we trained the ML classifiers. The expert group, including a medical doctor who specializes in EE diagnosis and a nutritionist who has profound knowledge in EE behavior, labeled a large portion of the data (5126 posts) as EE (coded as 1 ) or others (coded as 0) to train ML classifiers. The Dutch Eating Behavior Questionnaire, the EE scale (EES) [36], the extended version of EES [37], the revised Three-Factor Eating Questionnaire [37], and the Emotional Appetite Questionnaire [38] were mainly used as reference during the labeling process. The 2 experts independently labeled 120 randomly selected posts, then discussed the labels until they reached consensus. This 
process was repeated 5 times. The overall labeling process yielded a Cohen kappa coefficient of 0.85 . This process of human labeling was conducted based on previous works $[39,40]$.
As a result, 563 posts were labeled as EE posts and 4563 as others (Table 1).

Table 1. Total number of posts used in training machine learning classifiers.

\begin{tabular}{lll}
\hline Data & Emotional eating (coded as 1) & Others (coded as 0) \\
\hline Posts used for ML classifiers, $\mathbf{n}^{\text {a }}$ & & \\
Training set (9-fold validation) & 507 & 4107 \\
Test set & 56 & 456 \\
Total posts for ML & 563 & 4563 \\
Final posts classified by ML, $n$ & 26,154 & 159,796 \\
\hline
\end{tabular}

${ }^{\mathrm{a}} \mathrm{ML}$ : machine learning.

\section{Training Machine Learning Classifiers}

We trained 5 kinds of ML classifiers and compared their performance in detecting EE posts. Naive Bayes, decision tree, support vector machine, k-nearest neighbor algorithm, and stochastic gradient descent (SGD) were selected to classify EE posts. To account for the imbalance caused by a low proportion of EE posts in the dataset, we considered the accuracy score as well as precision, recall, and F1 scores to evaluate the performance of the models. Among the ML classifiers, the SGD classifier showed the best performance (see Table 2). The SGD classifier achieved the highest mean accuracy (0.90), precision (0.92), recall (0.91), and F1 score (0.91). K-fold cross-validation $(\mathrm{k}=0)$. A mean cross-validated area under curve (AUC) of 0.89 was obtained. Receiver operating characteristic curve represented the change of true positive rate and false positive rate, whereas the AUC represents the ML model. The SGD classifier labeled 26,154 EE posts and 194,435 corresponding comments.

Table 2. Performance of machine learning classifiers.

\begin{tabular}{lllll}
\hline Machine learning method & Precision & Recall & F1 score & Accuracy $(\%)$ \\
\hline Naive Bayes & 0.87 & 0.81 & 0.83 & 81 \\
Decision tree & 088 & 0.88 & 0.88 & 88 \\
Support vector machine & 0.90 & 0.91 & 0.90 & 91 \\
K-nearest neighbor & 0.86 & 0.88 & 0.87 & 83 \\
Stochastic gradient descent & 0.92 & 0.91 & 0.91 & 91 \\
\hline
\end{tabular}

\section{Preprocessing of Data and Topic Modeling With Latent Dirichlet Allocation}

To explore the online behavior patterns of emotional eaters, topic modeling process was conducted with LDA. For more advanced topic modeling, we preprocessed the EE posts classified with SGD before the LDA analysis. Natural language toolkit (NLTK) in Python was used for NLP. Preprocessing of data included the following 4 steps: (1) removing punctuation marks, (2) tokenizing and lemmatizing, (3) removing predefined stop words, and (4) performing term frequency-inverse document frequency (TF-IDF) vectorization. In step 3, removing stop words removes redundant and nonconsequential terms in the corpora. NLTK provides a built-in list of stop words, but we updated it for our research purposes. To better focus on the semantic aspects of the topics, we added auxiliary verbs and conjunctions that appeared repeatedly without particular meaning to the list of stop words (ie, "when," "be," "have," "not," "do," "so," "when," "would," "that," "can," and "more"). The modified list of stop words is on our Github page, with the codes of data analysis including ML classifier and LDA. This process was conducted under the supervision of experts who analyzed a subsample of terms that were considered for removal.
Finally, in step 4, the texts were converted into a term-document matrix where each word was assigned a weight using the TF-IDF weighing scheme. With these preprocessed data, we conducted the LDA topic modeling.

\section{Statistical Analysis of Feedback Patterns}

A total of number of 194,435 feedback comments on 26,154 posts were analyzed using statistical analysis. A chi-square test was conducted to distinguish the different proportions of 5 feedback topics in 4 types of EE posts.

\section{Results}

\section{Topics Related to Emotional Eating}

Throughout the LDA topic modeling analysis, EE posts were categorized into 4 topics: (1) EE 1, addressing feelings; (2) EE 2, sharing physical changes; (3) EE 3, sharing or asking for dietary information; and (4) EE 4, sharing dietary strategies (see Table 3). To minimize bias while categorizing the topics, the 4 topics were prudently chosen with iterative discussions among the experts, including a clinical doctor, a dietitian, and a nutritionist. All excerpts from the data were paraphrased and anonymized before being discussed. 
Table 3. Topics of emotional eating behavior and distinguishable linguistic differences.

\begin{tabular}{|c|c|c|c|}
\hline Number & Topic & Frequent words & Additional words found in the topic \\
\hline $\mathrm{EE}^{\mathrm{a}} 1$ & Addressing feelings & $\begin{array}{l}\text { Feel, good, love, really, depress, food, } \\
\text { hate, ever, much, change, and big }\end{array}$ & $\begin{array}{l}\text { Defeated, disgusting, hopeless, bad, full, shit, guilty, hungry, famished, } \\
\text { better, nice, and satisfying }\end{array}$ \\
\hline EE 2 & $\begin{array}{l}\text { Sharing physical } \\
\text { changes }\end{array}$ & $\begin{array}{l}\text { Weight, lose, lb, pound, gain, lose, } \\
\text { weight, get, diet, time, loss, exercise, } \\
\text { track, lose, and scale }\end{array}$ & $\begin{array}{l}\text { Shape, scalable value, nonscalable value, progress, accomplishment, reg- } \\
\text { ular, successful, courage, and back }\end{array}$ \\
\hline EE 3 & $\begin{array}{l}\text { Sharing or asking } \\
\text { for dietary informa- } \\
\text { tion }\end{array}$ & $\begin{array}{l}\text { Food, drink, meal, snack, pizza, } \\
\text { breakfast, sugar, work, chocolate, } \\
\text { fruit, chicken, soda, cream, chip, sal- } \\
\text { ad, ice, bread, and veggie }\end{array}$ & $\begin{array}{l}\text { Carbohydrate, fat, protein, ideas, triggers, advice, daily, nutrition, water, } \\
\text { experience, and favorite }\end{array}$ \\
\hline EE 4 & $\begin{array}{l}\text { Sharing dietary } \\
\text { strategies }\end{array}$ & $\begin{array}{l}\text { Binge, think, food, eat, emotion, } \\
\text { mental, help, binge eating, and control }\end{array}$ & $\begin{array}{l}\text { Keto, calorie in/calorie out, yoyoing, couch to } 5 \mathrm{~K}, 1200 \mathrm{cal} \text {, diet, protein, } \\
\text { extreme, and appetite }\end{array}$ \\
\hline
\end{tabular}

${ }^{\mathrm{a}}$ EE: emotional eating.

\section{User Behaviors Related to Emotional Eating Topic 1: Addressing Feelings}

Topic EE 1 mainly reflects the users' expressions of feelings toward specific eating behaviors or the food itself. The following words are sample contents analyzed based on our EE topic model: "feel," "depress," "good," "love," and "hate." For these words, the predicted probability of EE (pEE) topic 1 (pEE 1) among 4 EE topics based on the LDA model is $100 \%$, which means that nearly all the posts on the forum contained these words. As EE includes eating in response to any emotions, whether that be positive or negative, the appearance of positive words such as "good" and "love" in the list of frequent words is consistent with known behaviors of emotional eaters. We also discovered consistent use of words such as "defeated," "disgusting," and "hopeless." This indicates that EE behaviors of EE 1 require the most delicate support, as there is a high chance that the user is currently in a sensitive emotional state. Below are example sentences that represent EE 1 behavior. pEE 1 indicates how well the sentence represents the topic EE 1:

I sit here weeping and feeling defeated... (pEE
$I=0.99)$
I tossed nearly all of my candy stash into the trash
tonight. In fact, I threw away all my candies yesterday
as well, but I went to market to get more. I'm
disgusting and hopeless. ( $p E E I=0.98)$

\section{User Behaviors Related to Emotional Eating Topic 2:} Sharing Physical Changes

In topic EE 2, users shared their stories about physical changes. EE often leads to fluctuations in weight, and depending on what, when, and how much the users eat, the majority of posts in EE 2 elaborate on stories of these frequent weight changes. Linguistic features frequently used for weight units ("lb," "pound," "scale," and "weight") and the words that represent weight changes ("lose," "loss," "gain," "get," and "track") are on the list of frequent words. The following excerpt shows the unstable physical status of the forum user with EE behaviors. Many quotes from posts contain words in the list of additional words of EE 2 (Table 3) such as "kilogram," "crept up," and "level":
The first six months of my diet were not bad. I managed to stay under 100 kilograms, but I was determined to lose more weight. I'm awful and handling my appetite and my weight though. My weight kept creeping up and now, the last time I remember being under 100 is in February of this year. (pEE 2=0.96)

\section{User Behaviors Related to Emotional Eating Topic 3: Sharing or Asking for Dietary Information}

In topic EE 3, users shared or and asked for dietary information. In this topic, users mainly discussed the amount of food intake, contents of major nutrients, and calorie information. Thus, the majority of words on the list of frequent words are names of food (eg, "snack," "pizza," "chocolate," "fruit," "chicken," "salad," and "bread"). EE 3 behaviors are expressed as stories or lists, accompanied by the emotional status of the user. In the sample post below, the user describes his excessive eating habit as a stressful situation:

\section{I managed to clear half of a medium-sized pizza even though it wasn't even that good. After the first two slices, I was content, but within 5 minutes, I had to urge to eat more. I feel like I can't bear to leave food uneaten. I kept eating until I hit four slices and finally, $I$ restrained myself and put the other half in the freezer. ( $p E E$ 3=0.98)}

\section{User Behaviors Related to Emotional Eating Topic 4: Sharing Dietary Strategies}

In topic EE 4, users mainly shared their dietary strategies, including diet plans and feedback on specific dietary methodologies. Most users who posted about EE 4 continued to communicate their experiences and strategies with community members. We discovered many controversial and unverified strategies shared among the users (eg, extremely restrained eating and $1200 \mathrm{cal}$ diet). Frequent words in this topic seemed irrelevant to dietary strategies, but after thorough data exploration, we observed that the majority of users who posted about EE 4 questioned their own strategies and sought help or information from others. The following excerpt is a sample post for EE 4: 
Last year, I pushed myself really hard and placed myself on a 1200-calorie diet while working out 5 times a week. I was always hungry and in constant pain. I lost about 30 pounds. In May, I started the keto diet with light exercising such as biking and my weight is just falling off! I find the keto diet to be very easy for me. $(p E E 4=0.95)$

\section{Feedback Analysis}

In contrast to the post topics sorted based on the semantic elements of the content, feedback on the posts mainly relied on the syntax of the context. These elements reflect the linguistic characteristics of the feedback, which are meaningful information for determining the topics of the feedback. Similarly, we went through the topic modeling process without stemming out the pronouns, proper nouns, and interjections in the preprocessing stage. In the end, the topics of the feedback were categorized into 5 main topics, including (1) dietary information, (2) compliment, (3) consolation, (4) Reddit bot, and (5) health information. Frequent words and relevant examples are included in Table 4.

Table 4. Topics of feedback.

\begin{tabular}{|c|c|c|c|}
\hline Number & Topic & Frequent words & Quotes from online posts \\
\hline$E F^{\mathrm{a}} 1$ & Dietary information & $\begin{array}{l}\text { Eat, food, meal, drink, breakfast, } \\
\text { lunch, dinner, water, protein, cook, } \\
\text { sugar, and hungry }\end{array}$ & $\begin{array}{l}\text { "For a quick snack, I love to grab a stick of beef jerky from the convenience } \\
\text { store or dollar store. Lasts a while, is fairly cheap, has between 100-150 } \\
\text { calories, and fills me for a while" }\end{array}$ \\
\hline EF 2 & Compliment & $\begin{array}{l}\text { Amazing, good, people, work, gift, } \\
\text { great, job, fit, love, really, wow, con- } \\
\text { gratulation, and nice }\end{array}$ & "A nice change! Inspiration for people like me going through this! :D" \\
\hline EF 3 & Consolation & $\begin{array}{l}\text { Feel, hope, hard, lose, year, motivate, } \\
\text { lb, month, keep, think, life, and } \\
\text { change }\end{array}$ & $\begin{array}{l}\text { "I am right there with you...But whatever, tomorrow is a new day, approach } \\
\text { it just like you have been before this weekend. You know what you have } \\
\text { to do and you know you can do it because you have done it before. Just } \\
\text { put your head down and keep going!" }\end{array}$ \\
\hline $\mathrm{EF} 4$ & Reddit bot & $\begin{array}{l}\text { Post, question, answer, } / \mathrm{r} / \text { loseit, auto- } \\
\text { mated, contact moderator, guide, bot, } \\
\text { and thank }\end{array}$ & $\begin{array}{l}\text { "I am a bot, and this action was performed automatically. Please [contact } \\
\text { the moderators of this subReddit] (/message/compose/r/loseit) if you have } \\
\text { any questions or concerns." }\end{array}$ \\
\hline EF 5 & Health information & $\begin{array}{l}\text { Weight, calorie, scale, loss, diet, exer- } \\
\text { cise, muscle, body, deficit, count, } \\
\text { burn, lift, and measure }\end{array}$ & $\begin{array}{l}\text { "Alcohol can dehydrate you in the very short term which will show up as } \\
\text { weight loss on the scale, but typically a body reacts by then retaining even } \\
\text { more water until it feels that it is sufficiently hydrated." }\end{array}$ \\
\hline
\end{tabular}

${ }^{\mathrm{a}} \mathrm{EF}$ : feedback on emotional eating behavior

\section{Feedback Distribution Based on Emotional Eating Topics}

The proportions of feedback topic distribution differed greatly depending on the topic of the post (Multimedia Appendix 1). Table 5 lists the dominant EF topic for each EE topic. A chi-square test of feedback topics among the 4 EE behaviors revealed statistically significant results. For the posts addressing feelings (EE 1), feedback sharing dietary intake (EF 1) were dominant. EE posts regarding physical changes (EE 2) were accompanied by compliments (EF 2) on daily achievements. EE posts sharing or asking for dietary information (EE 3) collected the most significant proportion of feedback with consolation. EE posts sharing dietary strategies (EE 4) were followed by feedback with health-related information.

Table 5. Dominant feedback topic on each emotional eating-related behavior topic.

\begin{tabular}{|c|c|c|c|c|}
\hline $\mathrm{EE}^{\mathrm{a}}$ topic & Dominant $\mathrm{EF}^{\mathrm{b}}$ topic & Ratio of feedback dominance & Chi-square value $(d f)$ & $P$ value \\
\hline EE 1: addressing feeling & EF 1: dietary information & 0.49 & $28328(4)$ & $<.001$ \\
\hline EE 2: sharing physical changes & EF 2: compliment & 0.46 & $19133(4)$ & $<.001$ \\
\hline $\begin{array}{l}\text { EE 3: sharing or asking for dietary } \\
\text { intake }\end{array}$ & EF 3: consolation & 0.34 & $1813.2(4)$ & $<.001$ \\
\hline EE 4: sharing dietary strategies & EF 5: health information & 0.30 & $3181.1(4)$ & $<.001$ \\
\hline
\end{tabular}

${ }^{\mathrm{a}} \mathrm{EE}$ : emotional eating.

${ }^{\mathrm{b}} \mathrm{EF}$ : feedback on emotional eating behavior. 


\section{Discussion}

\section{Data-Driven Approach for the Determination of Users' Emotional Eating Behavior}

This study introduces a data-driven approach for determining the abnormal behaviors (ie, EE) of social forum users. EE-related data were classified with our trained SGD classifier, and 4 types of EE behaviors and 5 types of feedback were distinguished with the LDA topic modeling method. The proportions of the feedback topics significantly differed for each EE behavior topic.

\section{Posts Addressing Feelings}

Previous studies have proven that both positive and negative emotions can lead to EE behaviors [3,36,41]. According to our Table 3, there were many words related to feelings such as depression, anger, and joy. Depression is an incessantly occurring feeling before and after EE [41]. Emotions such as anger, fear, sadness, and joy often last a long time and linger $[42,43]$, which could explain the frequent usage of these words in posts that address feelings. Low-arousal states such as boredom and depression are often associated with inhibition of food intake, especially compared with high-arousal states such as tension and fear. However, depression was at the top of the list of frequent words for emotional eaters, so we inferred that depressive feelings frequently occur with EE behavior $[2,41]$. This accumulation of emotional data allows us to analyze what provokes EE behavior and guides us to implementing a feedback function as a personalized support system (PSS).

\section{Posts Sharing Physical Changes}

Users with EE behaviors share their physical changes not only as a way to monitor themselves but also with expectations of social support according to our data. Thus, it is not surprising that the most dominant feedback on this type of post is a compliment. We believe that sharing physical changes through the online community can be a useful tactic for emotional eaters to get encouragement. In addition, we can track the users' health status and design a PSS that provides behavioral guidelines in response to the users' physical changes.

Interestingly, some of the people who showed the behavior of sharing their physical changes had obsessive characteristics (eg, weighing too often and reacting sensitively to small changes in the body). This obsessive nature often forces them to implement an overly strict plan that restrains eating, which leaves them more vulnerable to EE in response to stressful situations [44,45]. Therefore, the PSS could include a warning that extreme dietary restrictions may interfere with long-term dietary management and may even interfere with weight control.

\section{Posts Sharing or Asking for Dietary Information}

It is not easy to predict the dietary patterns of emotional eaters because EE occurs unpredictably. In the field of medicine or nutrition, the food frequency questionnaire or 24-hour recall methods are used to track dietary information [46]. However, the dietary information collected through these methods often lack representation because of insufficient data or problems with memory retrieval [46]. Researchers have tried to improve these dietary tracking methods with direct inputs of food intake, but these methods are cumbersome for long-term use [47]. Many social forum users share their dietary information through social media such as Twitter and Instagram [48,49]. Social media offers users with better and easier experiences in terms of recording dietary information. Therefore, this study takes advantage of the opportunity to use the dietary information on online communities for analysis.

\section{Posts Sharing Dietary Strategies}

Through posts sharing dietary strategies, we were able to identify diets such as calories in-calories out, keto, and 1200 $\mathrm{kcal}$ diet strategies. We also discovered that these dietary strategies can pose a serious health threat or aggravate EE behavior among emotional eaters [5]. As these strategies are contentious, the feedback sharing health information (EF 5) was the majority of the feedback on posts about dietary strategies. People with EE behavior should abstain from following controversial dietary strategies, but if they were to adhere to such diet, they should be well aware of the side effects such as appetite fluctuations and malnutrition [50]. It is critical that reliable information is shared among those with abnormal health behaviors as false information posted on an online community can cause damage to a large group of people. However, studies have shown that $89 \%$ of health-related information provided on online medical forums was written by people without professional experience [51,52] or medical practices [53,54]. By providing tailored information complied by experts and reliable references, PSS can be a crucial solution to false health information and discussions in the online community.

\section{Feedback Topics}

Previous studies have highlighted the importance of feedback on health-related behaviors [55]. Feedback analysis revealed that feedback differed greatly depending on the EE behavior patterns. Feedback on EE behavior (EF) were categorized into 5 topics by experts based on LDA results (see Table 3). Dietary information in EF 1 describes not only personal dietary experiences but also dietary facts, both with and without proper reference. Compliments in EF 2 is one of the most effective forms of feedback [55] that keeps users motivated for a long period [56]. Consolation in EF 3 differs from a compliment as consolation feedback mostly appears on the posts of negative status [57] and aims to uplift those in challenging circumstances. Bot-generated feedback (EF 4) was easily distinguishable from other feedback, so the LDA model was able to classify them with high performance. EF 5 contains health-related information.

\section{Latent Dirichlet Allocation Topic Model as a User Study Method Before Designing a Support System for Users With Abnormal Behaviors}

One of the challenges of user study comes from repeated experiments without proper understanding of user characteristics and preparation procedures. Thus, a long-standing investigation of previous studies and a pilot study attempt to overcome this challenge. Nevertheless, exploration of user characteristics through these methods still faces limitations as pilot studies can only reflect the characteristics of a limited subgroup. This gap 
may be ignored in small group studies, but it will lead to a significant difference in large studies.

Although the topic modeling method does not completely overcome the limitations of prior methods, it can be useful to user groups with abnormal behaviors. In addition, through topic modeling, we were able to identify prior systems that users with EE relied on (eg, MyFitnessPal for physical changes [EE 2] and total daily energy expenditure for dietary information [EE 3]). Therefore, topic modeling is applicable as a good alternative data-driven qualitative method for designing a support system for specific user group.

From the HCI-based approach, we propose a systematic design for a PSS that provides reliable and confidential information just-in-time. Although there are many health-specific websites that offer focused information verified by professionals, many online users prefer to discuss health issues on social media [58]. Therefore, it is also necessary to conduct additional research on designing social media-based PSS as a way to support the users on social media.

Previous studies demonstrate the need of personalized interventions that are customized and predetermined $[59,60]$. This study contributes to the medical field by detecting and analyzing abnormal behavior patterns of emotional eaters who are in need of PSS design. In addition, the ML classifier used in this study is highly applicable in the PSS development process as it can detect EE-related posts just-in-time with high accuracy.

\section{Limitations}

This study was the first step toward understanding abnormal behavior patterns of EE to design PSS. Thus, there were several limitations that can be improved in the future works.

First, although we used a systematic topic modeling method in a data-driven approach to explore EE behavior, the categories of the topics were determined empirically by humans. In this study, we gathered domain experts to discuss their opinions and relied on their decisions to compensate for the limitations of methods. The researchers did not intervene in the decision-making process after providing the experts with sufficient explanations on the topic modeling method. Further research on establishing a standard procedure for feature selection, especially the number of categories of post topics, is necessary. In this study, EE behaviors were only sorted into 4 different categories to observe the macroscopic patterns of behaviors. However, for a more advanced system design, EE-related posts should be further distinguished into smaller subcategories with clear classification criteria.

Next, we discussed the need for PSS based on the results of our study. However, before we establish a support system, we need to investigate how users will react to bot intervention and whether this intervention will increase the users' engagement in the community. The roles of community bot have been debated recently among the HCI community [61-63], but further research, specifically regarding users with abnormal behaviors, needs to be conducted.

Finally, as the EE data were collected from a single Reddit subcommunity dealing with weight management, there can be a bias that classifies the posts as EE behavior. However, we have attempted to minimize this bias through sufficient discussion among experts during the process of data classification and excluded posts that were not relevant to EE.

\section{Conclusions}

This work investigates the behavior and feedback patterns of Reddit users with EE behaviors. First, we analyzed the data classified with our ML classifier to detect EE behaviors in the online community. Second, we analyzed EE behaviors and feedback topics with LDA. EE behaviors were classified into 4 main topics: addressing feelings, sharing physical changes, sharing dietary information, and sharing strategies to control EE. EF behaviors were classified into 5 topics: dietary information, compliment, consolation, Reddit bot, and health information. Our work significantly extended prior user studies on abnormal behavior patterns in the field of digital medicine research. Furthermore, our results provide new insights for designing a PSS for users with abnormal behaviors. The main contributions of this work are as follows:

- ML classifier with high accuracy that collects behavioral data of posts demonstrating EE behaviors,

- Possibility of the LDA topic model as an exploratory behavioral research method for classifying abnormal behaviors in the field of digital medicine,

- Opportunities for PSS implementation to help emotional eaters.

\section{Acknowledgments}

This work was supported by the Ministry of Education of the Republic of Korea and the National Research Foundation of Korea (NRF-2018S1A3A2075114).

\section{Conflicts of Interest}

None declared.

\section{Multimedia Appendix 1}

Feedback topic proportion depending on the post topic.

[PNG File, 75 KB-Multimedia Appendix 1]

\section{References}


1. Keski-Rahkonen A, Mustelin L. Epidemiology of eating disorders in Europe: prevalence, incidence, comorbidity, course, consequences, and risk factors. Curr Opin Psychiatry 2016 Nov;29(6):340-345. [doi: 10.1097/YCO.0000000000000278] [Medline: 27662598]

2. van Strien T. Causes of emotional eating and matched treatment of obesity. Curr Diab Rep 2018 Apr 25;18(6):35 [FREE Full text] [doi: 10.1007/s11892-018-1000-x] [Medline: 29696418]

3. Barrada JR, van Strien T, Cebolla A. Internal Structure and Measurement Invariance of the Dutch Eating Behavior Questionnaire (DEBQ) in a (Nearly) Representative Dutch Community Sample. Eur Eat Disord Rev 2016 Nov;24(6):503-509. [doi: 10.1002/erv.2448] [Medline: 27075404]

4. van Strien T, Schippers GM, Cox WM. On the relationship between emotional and external eating behavior. Addict Behav 1995;20(5):585-594. [doi: 10.1016/0306-4603(95)00018-8] [Medline: 8712056]

5. van Strien T, Frijters JE, Bergers GP, Defares PB. The Dutch Eating Behavior Questionnaire (DEBQ) for assessment of restrained, emotional, and external eating behavior. Int J Eat Disord 1986;5(2):295-315. [doi: 10.1002/1098-108x(198602)5:2<295::aid-eat2260050209>3.0.co;2-t]

6. Cheng J, Bernstein M, Danescu-Niculescu-Mizil C, Leskovec J. Anyone Can Become a Troll: Causes of Trolling Behavior in Online Discussions. In: Proceedings of the 2017 ACM Conference on Computer Supported Cooperative Work and Social Computing. USA: ACM; 2017 Presented at: CSCW'17; Oregon, Portland, USA; February 25 - March 1, 2017 p. $1217-1230$. [doi: $\underline{10.1145 / 2998181.2998213}$ ]

7. DeVito MA, Walker AM, Birnholtz J. 'Too Gay for Facebook': Presenting LGBTQ+ identity throughout the personal social media ecosystem. Proc ACM Hum-Comput Interact 2018;2(44):1-23. [doi: 10.1145/3274313]

8. Liu H, Salerno J, Young MJ. Social Computing and Behavioral Modeling. New York, NY: Springer; 2009.

9. Panger G. People tend to wind down, not up, when they browse social media. Proc ACM Hum-Comput Interact 2018;2(133):1-29. [doi: 10.1145/3274402]

10. Rho EH, Mark G, Mazmanian M. Fostering civil discourse online: linguistic behavior in comments of \#MeToo articles across political perspectives. Proc ACM Hum-Comput Interact 2018;2(147):1-28. [doi: 10.1145/3274416]

11. Fischer E, Reuber AR. Social interaction via new social media: (How) can interactions on Twitter affect effectual thinking and behavior? J Bus Ventur 2011;26(1):1-18. [doi: 10.1016/j.jbusvent.2010.09.002]

12. Kim C, Yang SU. Like, comment, and share on Facebook: how each behavior differs from the other. Public Relat Rev 2017;43(2):441-449. [doi: 10.1016/j.pubrev.2017.02.006]

13. Yang T. The decision behavior of Facebook users. J Comput Inf Syst 2012;52(3):50-59 [FREE Full text]

14. Appel H, Gerlach AL, Crusius J. The interplay between Facebook use, social comparison, envy, and depression. Curr Opin Psychol 2016;9:44-49. [doi: 10.1016/j.copsyc.2015.10.006]

15. Greene JA, Choudhry NK, Kilabuk E, Shrank WH. Online social networking by patients with diabetes: a qualitative evaluation of communication with Facebook. J Gen Intern Med 2011 Mar;26(3):287-292 [FREE Full text] [doi:

10.1007/s11606-010-1526-3] [Medline: 20945113]

16. Jelenchick LA, Eickhoff JC, Moreno MA. 'Facebook depression?' social networking site use and depression in older adolescents. J Adolesc Health 2013 Jan;52(1):128-130. [doi: 10.1016/j.jadohealth.2012.05.008] [Medline: 23260846]

17. Shen J, Brdiczka O, Liu J. A study of Facebook behavior: what does it tell about your Neuroticism and Extraversion? Comput Human Behav 2015;45:32-38 [FREE Full text] [doi: 10.1016/j.chb.2014.11.067]

18. Joshi DJ, Supekar N, Chauhan R, Patwardhan MS. Modeling and Detecting Change in User Behavior Through His Social Media Posting Using Cluster Analysis. In: Proceedings of the Fourth ACM IKDD Conferences on Data Sciences. USA:

ACM; 2017 Presented at: CODS'17; March 9 - 11, 2017; Chennai, India p. 1-9. [doi: 10.1145/3041823.3041830]

19. Toseeb U, Inkster B. Online social networking sites and mental health research. Front Psychiatry 2015;6:36 [FREE Full text] [doi: 10.3389/fpsyt.2015.00036] [Medline: 25814958]

20. Surian D, Nguyen DQ, Kennedy G, Johnson M, Coiera E, Dunn AG. Characterizing Twitter discussions about HPV vaccines using topic modeling and community detection. J Med Internet Res 2016 Aug 29;18(8):e232 [FREE Full text] [doi: 10.2196/jmir.6045] [Medline: 27573910]

21. Benhammou Y, Tabik S, Achchab B, Herrera F. A First Study Exploring the Performance of the State-of-the Art CNN Model in the Problem of Breast Cancer. In: Proceedings of the International Conference on Learning and Optimization Algorithms: Theory and Applications. USA: ACM; 2018 Presented at: LOPAL'18; May 2 - 5, 2018; Rabat, Morocco p. 1-6. [doi: 10.1145/3230905.3230940]

22. Cui X, Yang N, Wang Z, Hu C, Zhu W, Li H, et al. Chinese social media analysis for disease surveillance. Pers Ubiquit Comput 2015;19:1125-1132. [doi: 10.1007/s00779-015-0877-5]

23. de Choudhury CM, Kiciman E, Dredze M, Coppersmith G, Kumar M. Discovering Shifts to Suicidal Ideation from Mental Health Content in Social Media. In: Proceedings of the 2016 CHI Conference on Human Factors in Computing Systems. USA: ACM; 2016 Presented at: CHI'16; May 7 - 12, 2016; California, San Jose, USA p. 2098-2110. [doi: $10.1145 / 2858036.2858207]$

24. Linke SE, Dunsiger SI, Gans KM, Hartman SJ, Pekmezi D, Larsen BA, et al. Association between physical activity intervention website use and physical activity levels among Spanish-speaking Latinas: randomized controlled trial. J Med Internet Res 2019 Jul 24;21(7):e13063 [FREE Full text] [doi: 10.2196/13063] [Medline: $\underline{\text { 31342902] }}$ 
25. McLennan S. Quantitative ratings and narrative comments on Swiss physician rating websites: frequency analysis. J Med Internet Res 2019 Jul 26;21(7):e13816 [FREE Full text] [doi: 10.2196/13816] [Medline: $\underline{\text { 31350838] }}$

26. Liu S, Zhu M, Yu DJ, Rasin A, Young SD. Using real-time social media technologies to monitor levels of perceived stress and emotional state in college students: a web-based questionnaire study. JMIR Ment Health 2017 Jan 10;4(1):e2 [FREE Full text] [doi: 10.2196/mental.5626] [Medline: 28073737]

27. Moreno MA, Jelenchick LA, Egan KG, Cox E, Young H, Gannon KE, et al. Feeling bad on Facebook: depression disclosures by college students on a social networking site. Depress Anxiety 2011 Jun;28(6):447-455 [FREE Full text] [doi: 10.1002/da.20805] [Medline: 21400639]

28. Park M, McDonald DW, Cha M. Perception Differences between the Depressed and Non-Depressed Users in Twitter. In: Proceedings of the Seventh International AAAI Conference on Weblogs and Social Media. 2013 Presented at: ICWSM-13; July 8-11, 2013; Cambridge, Massachusetts.

29. Wolf M, Sedway J, Bulik CM, Kordy H. Linguistic analyses of natural written language: unobtrusive assessment of cognitive style in eating disorders. Int J Eat Disord 2007 Dec;40(8):711-717. [doi: 10.1002/eat.20445] [Medline: 17683092]

30. Park A, Conway M. Harnessing Reddit to understand the written-communication challenges experienced by individuals with mental health disorders: analysis of texts from mental health communities. J Med Internet Res 2018 Apr 10;20(4):e121 [FREE Full text] [doi: 10.2196/jmir.8219] [Medline: 29636316]

31. Blei DM, Ng AY, Jordan M. Latent Dirichlet Allocation. J Mach Learn Res 2003;3:601-608 [FREE Full text]

32. Crossno PJ, Wilson AT, Shead TM, Dunlavy DM. TopicView: Visually Comparing Topic Models of Text Collections. In: Proceedings of the 2011 IEEE 23rd International Conference on Tools with Artificial Intelligence. Palm Beach County, Florida, US: IEEE; 2011 Presented at: ICTAI'11; November 7-9, 2011; Boca Raton, FL, USA. [doi: 10.1109/ictai.2011.162]

33. Řehůřek R, Sojka P. Software Framework for Topic Modelling with Large Corpora. In: Proceedings of LREC 2010 Workshop New Challenges for NLP Frameworks. Valletta, Malta: University of Malta; 2010 Presented at: LREC'10; May 17-23, 2010; Malta.

34. Kozlowski AC, Taddy M, Evans JA. arXiv e-Print archive. 2018. The Geometry of Culture: Analyzing Meaning through Word Embeddings URL: https://arxiv.org/abs/1803.09288 [accessed 2020-01-15]

35. Pappa GL, Cunha TO, Bicalho PV, Ribeiro A, Silva AP, Meira W, et al. Factors associated with weight change in online weight management communities: a case study in the LoseIt Reddit community. J Med Internet Res 2017 Jan 16;19(1):e17 [FREE Full text] [doi: 10.2196/jmir.5816] [Medline: 28093378]

36. Arnow B, Kenardy J, Agras WS. The Emotional Eating Scale: the development of a measure to assess coping with negative affect by eating. Int J Eat Disord 1995 Jul;18(1):79-90. [doi: 10.1002/1098-108x(199507)18:1<79::aid-eat2260180109>3.0.co;2-v] [Medline: 7670446$]$

37. Kenardy J, Butler A, Carter C, Moor S. Eating, mood, and gender in a noneating disorder population. Eat Behav 2003 Aug;4(2):149-158. [doi: 10.1016/S1471-0153(03)00019-9] [Medline: 15000978]

38. Wallis DJ, Hetherington MM. Emotions and eating. Self-reported and experimentally induced changes in food intake under stress. Appetite 2009 Apr;52(2):355-362. [doi: 10.1016/j.appet.2008.11.007] [Medline: 19071171]

39. Andalibi N, Haimson OL, de Choudhury M, Forte A. Understanding Social Media Disclosures of Sexual Abuse Through the Lenses of Support Seeking and Anonymity. In: Proceedings of the 2016 CHI Conference on Human Factors in Computing Systems. New York, New York, USA: ACM Press; 2016 Presented at: CHI'16; May 7 - 12, 2016; California, San Jose, US p. 3906-3918. [doi: 10.1145/2858036.2858096]

40. Andalibi N, Ozturk P, Forte A. Sensitive Self-disclosures, Responses, and Social Support on Instagram: The Case of \#Depression. In: Proceedings of the 2017 ACM Conference on Computer Supported Cooperative Work and Social Computing. New York, New York, USA: ACM Press; 2017 Presented at: CSCW'17; February 25 - March 1, 2017; Oregon, Portland, USA p. 1485-1500. [doi: 10.1145/2998181.2998243]

41. Kenardy J, Arnow B, Agras WS. The aversiveness of specific emotional states associated with binge-eating in obese subjects. Aust N Z J Psychiatry 1996 Dec;30(6):839-844. [doi: 10.3109/00048679609065053] [Medline: 9034475]

42. Ekman P. An argument for basic emotions. Cogn Emot 1992;6(3-4):169-200. [doi: 10.1080/02699939208411068]

43. Frijda NH. Moods, emotion episodes, and emotions. In: Barrett LF, Lewis M, Haviland-Jones JM, editors. Handbook of Emotions. New York City: Guilford Press; 1993:381-403.

44. Heatherton T, Herman C, Polivy J. Effects of physical threat and ego threat on eating behavior. J Pers Soc Psychol 1991 Jan;60(1):138-143. [doi: 10.1037//0022-3514.60.1.138] [Medline: 1995833]

45. Robbins TW, Fray PJ. Stress-induced eating: fact, fiction or misunderstanding? Appetite 1980;1(2):103-133. [doi: 10.1016/s0195-6663(80)80015-8]

46. Bingham SA, Gill C, Welch A, Day K, Cassidy A, Khaw KT, et al. Comparison of dietary assessment methods in nutritional epidemiology: weighed records v. $24 \mathrm{~h}$ recalls, food-frequency questionnaires and estimated-diet records. Br J Nutr 1994 Oct;72(4):619-643. [doi: 10.1079/bjn19940064] [Medline: 7986792]

47. Hakobyan L, Lumsden J, Shaw R, O?Sullivan D. A Longitudinal Evaluation of the Acceptability and Impact of a Diet Diary App for Older Adults with Age-related Macular Degeneration. In: Proceedings of the 18th International Conference on Human-Computer Interaction with Mobile Devices and Services. USA: ACM; 2016 Presented at: MobileHCI'16; September 6 - 9, 2016; Florence, Italy p. 124-134. [doi: 10.1145/2935334.2935356] 
48. Abbar S, Mejova Y, Weber I. You Tweet What You Eat: Studying Food Consumption Through Twitter. In: Proceedings of the 33rd Annual ACM Conference on Human Factors in Computing Systems. USA: ACM; 2015 Presented at: CHI'15; April 18 - 23, 2015; Seoul, Republic of Korea p. 3197-3206. [doi: 10.1145/2702123.2702153]

49. Chung CF, Agapie E, Schroeder J, Mishra S, Fogarty J, Munson SA. When Personal Tracking Becomes Social: Examining the Use of Instagram for Healthy Eating. In: Proceedings of the 2017 CHI Conference on Human Factors in Computing Systems. USA: ACM; 2017 Presented at: CHI'17; May 6 - 11, 2017; Colorado, Denver, USA p. 1674-1687. [doi: 10.1145/3025453.3025747]

50. Dirks AJ, Leeuwenburgh C. Caloric restriction in humans: potential pitfalls and health concerns. Mech Ageing Dev 2006 Jan;127(1):1-7. [doi: 10.1016/j.mad.2005.09.001] [Medline: 16226298]

51. Culver JD, Gerr F, Frumkin H. Medical information on the internet: a study of an electronic bulletin board. J Gen Intern Med 1997 Aug;12(8):466-470 [FREE Full text] [doi: 10.1046/j.1525-1497.1997.00084.x] [Medline: 9276651]

52. Eastin MS. Credibility assessments of online health information: the effects of source expertise and knowledge of content. J Comput Mediat Commun 2001;6(4). [doi: 10.1111/j.1083-6101.2001.tb00126.x]

53. Dudley T, Falvo D, Podell R, Renner J. The informed patient poses a different challenge. Patient Care 1996;30(16):128 [FREE Full text]

54. Lindberg DA, Humphreys BL. Medicine and health on the internet: the good, the bad, and the ugly. J Am Med Assoc 1998 Oct 21;280(15):1303-1304. [doi: 10.1001/jama.280.15.1303] [Medline: 9794299]

55. Seyff N, Ollmann G, Bortenschlager M. AppEcho - A User-driven, In Situ Feedback Approach for Mobile Platforms and Applications. In: Proceedings of the 1st International Conference on Mobile Software Engineering and Systems. 2014 Presented at: MOBILESoft'2014; June 2 - 3, 2014; Hyderabad, India p. 99-108. [doi: 10.1145/2593902.2593927]

56. Thornton PL, Kieffer EC, Salabarría-Peña Y, Odoms-Young A, Willis SK, Kim H, et al. Weight, diet, and physical activity-related beliefs and practices among pregnant and postpartum Latino women: the role of social support. Matern Child Health J 2006 Jan;10(1):95-104. [doi: 10.1007/s10995-005-0025-3] [Medline: 16534660]

57. Swann WB, Predmore SC. Intimates as agents of social support: sources of consolation or despair? J Pers Soc Psychol 1985 Dec;49(6):1609-1617. [doi: 10.1037//0022-3514.49.6.1609] [Medline: 4087141]

58. Antin J, de Sa M, Churchill E. Local Experts and Online Review Sites. In: Proceedings of the ACM 2012 conference on Computer Supported Cooperative Work Companion. USA: ACM; 2012 Presented at: CSCW'12; February 11 - 15, 2012 ; Washington, Seattle, USA p. 55-58. [doi: 10.1145/2141512.2141541]

59. Bezemer T, de Groot MC, Blasse E, Ten Berg MJ, Kappen TH, Bredenoord AL, et al. A Human(e) Factor in Clinical Decision Support Systems. J Med Internet Res 2019 Mar 19;21(3):e11732 [FREE Full text] [doi: 10.2196/11732] [Medline: 30888324]

60. Moreno MA, D'Angelo J. Social media intervention design: applying an affordances framework. J Med Internet Res 2019 Mar 26;21(3):e11014 [FREE Full text] [doi: 10.2196/11014] [Medline: 30912754]

61. Krafft PM, Macy M, Pentland A. Bots as Virtual Confederates: Design and Ethics. In: Proceedings of the 2017 ACM Conference on Computer Supported Cooperative Work and Social Computing. USA: ACM; 2017 Presented at: CSCW'17; February 25 - March 1, 2017; Oregon, Portland, USA p. 183-190. [doi: 10.1145/2998181.2998354]

62. Long K, Vines J, Sutton S, Brooker P, Feltwell T, Kirman B, et al. 'Could You Define That in Bot Terms'?: Requesting, Creating and Using Bots on Reddit. In: Proceedings of the 2017 CHI Conference on Human Factors in Computing Systems. New York, NY. USA: ACM; 2017 Presented at: CHI'17; May 6 - 11, 2017; Colorado, Denver, USA p. 3488-3500. [doi: 10.1145/3025453.3025830]

63. Seering J, Flores JP, Savage S, Hammer J. The social roles of bots: evaluating impact of bots on discussions in online communities. Proc ACM Hum-Comput Interact 2018;2(157):1-29. [doi: 10.1145/3274426]
Abbreviations
AUC: area under curve
EE: emotional eating
EES: emotional eating scale
EF: feedback on EE behavior
HCI: human-computer interaction
LDA: Latent Dirichlet Allocation
NLP: natural language processing
NLTK: natural language toolkit
pEE: probability of emotional eating
PSS: personalized support system
SGD: stochastic gradient descent
TF-IDF: term frequency-inverse document frequency 
Edited by G Eysenbach; submitted 31.07.19; peer-reviewed by CA Almenara, D Carvalho; comments to author 15.10.19; revised version received 08.12.19; accepted 16.12.19; published 31.03.20

Please cite as:

Hwang Y, Kim HJ, Choi HJ, Lee J

Exploring Abnormal Behavior Patterns of Online Users With Emotional Eating Behavior: Topic Modeling Study

$J$ Med Internet Res 2020;22(3):e15700

URL: http://www.jmir.org/2020/3/e15700/

doi: $10.2196 / 15700$

PMID: 32229461

(CYoujin Hwang, Hyung Jun Kim, Hyung Jin Choi, Joonhwan Lee. Originally published in the Journal of Medical Internet Research (http://www.jmir.org), 31.03.2020. This is an open-access article distributed under the terms of the Creative Commons Attribution License (https://creativecommons.org/licenses/by/4.0/), which permits unrestricted use, distribution, and reproduction in any medium, provided the original work, first published in the Journal of Medical Internet Research, is properly cited. The complete bibliographic information, a link to the original publication on http://www.jmir.org/, as well as this copyright and license information must be included. 